\title{
T-cell tailored single-cell RNA barcoding and sequencing (tSCRB-seq)
}

\section{Kristiyan Kanev ( $\boldsymbol{D}$ kanev@wzw.tum.de )}

Division of Animal Physiology and Immunology, School of Life Sciences Weihenstephan, Technical University of Munich, 85354 Freising, Germany https://orcid.org/0000-0002-2705-9307

\section{Patrick Roelli}

Division of Animal Physiology and Immunology, School of Life Sciences Weihenstephan, Technical University of Munich, 85354 Freising, Germany

\section{Ming Wu}

Division of Animal Physiology and Immunology, School of Life Sciences Weihenstephan, Technical University of Munich, 85354 Freising, Germany

\section{Christine Wurmser}

Division of Animal Breeding, School of Life Sciences Weihenstephan, Technical University of Munich, 85354 Freising,

\section{Germany}

\section{Mauro Delorenzi}

BCF, Swiss Institute of Bioinformatics, University of Lausanne 1015 Lausanne, Switzerland

\section{Michael W. Pfaffl}

Division of Animal Physiology and Immunology, School of Life Sciences Weihenstephan, Technical University of Munich, 85354 Freising, Germany

\section{Dietmar Zehn ( $\nabla$ dietmar.zehn@tum.de )}

Division of Animal Physiology and Immunology, School of Life Sciences Weihenstephan, Technical University of Munich, 85354 Freising, Germany

\section{Method Article}

Keywords: primary T cells, single-cell RNA sequencing

Posted Date: January 8th, 2021

DOl: https://doi.org/10.21203/rs.3.pex-1289/v1

License: (c) (i) This work is licensed under a Creative Commons Attribution 4.0 International License. Read Full License 


\section{Abstract}

tSCBR-seq is a T-cell tailored plate-based single-cell RNA sequencing protocol with superior mRNA capturing efficacy and dynamic range of gene detection. This was achieved through a series of chemical modifications of the original SCBR-seq protocol published by Soumilon and colleagues [1]. The performance of the protocol was also demonstrated in the article "Proliferation-competent Tcf1 + CD8 T cells in dysfunctional populations are CD4 T cell help independent" [2].

\section{Introduction}

\section{Reagents}

RNaseZap - RNase Decontamination Solution

AM9780, Invitrogen

FrameStar 96-Well Semi-Skirted PCR Plate, low binding

4ti-LB0770/C, 4titude

Buffer TCL*

1031576, Qiagen

2-Mercaptoethanol, molecular biology grade

63689-25ML-F, Sigma

Adhesive PCR Foil Seals**

4TI-0550, 4titude

Dry ice

various vendors

RNAClean XP beads*

A63987, Beckman Coulter

Absolute Ethanol, molecular biology grade

BP2818500, Fisher BioReagents

Page 2/30 
$25 \mathrm{ml}$ Reagent Reservoir, PS, sterile

various vendors

NxGen RNAse Inhibitor

30281-2, Lucigen

Maxima H Minus Reverse Transcriptase

EP0753, Thermo Fisher Scientific

Advantage ${ }^{\circledR}$ UltraPure PCR Deoxynucleotide Mix (10 mM each dNTP)*

639125, Takara

KAPA HiFi HotStart ReadyMix*

7958935001, KAPA Biosystems

AMPure XP*

A63881, Beckman Coulter

High Sensitivity DNA Kit (Chips \& Reagents)

5067-4626, Agilent

Nextera XT DNA Library Preparation Kit*

FC-131-1024, Illumina

Water, molecular biology grade

various vendors

Filter tips $(10 \mu l, 20 \mu l$ and $1000 \mu l)$ 
various vendors

$1.5 \mathrm{ml}$ DNA LoBind Tubes

022431021, Eppendorf

2.0 ml DNA LoBind Tubes

022431048, Eppendorf

$0.2 \mathrm{ml}$ 8-Tube Strips (with attached caps)

various vendors

PCR Seal

4ti-0500, 4titude

PCR Seal perforated for tearing into part plates or 8 well strips

4ti-0500/8, 4titude

Segmented PCR plates, $3 \times 8$ wells

4ti-0750/24, 4titude

Filter pipette tips

various vendors

* Do not replace.

** Make sure it remains sealed upon plate storage at $-70^{\circ} \mathrm{C}$.

\section{Primers:}

SCRB-seq Template Switching Oligo (TSO)

iC-iG-iCACACTCTTTCCCTACACGACGCrGrGrG

Page $4 / 30$ 
SCRB-seq SMART PCR Primer

/5Biosg/ACACTCTTTCCCTACACGACGC

/5Biosg/ denotes a 5' biotin; HLPC purification

SCRB-seq custom N5 primer

AATGATACGGCGACCACCGAGATCTACACTCTTTCCCTACACGACGCTCTTCCG*A*T*C*T*

* denotes a phosphorothioate bond; HLPC purification

Nextera N701 Primer

CAAGCAGAAGACGGCATACGAGATTCGCCTTAGTCTCGTGGGCTCGG

HLPC purification

Nextera N702 Primer

CAAGCAGAAGACGGCATACGAGATCTAGTACGGTCTCGTGGGCTCGG

HLPC purification

Nextera N703 Primer

CAAGCAGAAGACGGCATACGAGATTTCTGCCTGTCTCGTGGGCTCGG

HLPC purification

Nextera N704 Primer

CAAGCAGAAGACGGCATACGAGATGCTCAGGAGTCTCGTGGGCTCGG

HLPC purification

Nextera N705 Primer

CAAGCAGAAGACGGCATACGAGATAGGAGTCCGTCTCGTGGGCTCGG

HLPC purification 
Nextera N706 Primer

CAAGCAGAAGACGGCATACGAGATCATGCCTAGTCTCGTGGGCTCGG

HLPC purification

Nextera N707 Primer

CAAGCAGAAGACGGCATACGAGATGTAGAGAGGTCTCGTGGGCTCGG

HLPC purification

Nextera N708 Primer

CAAGCAGAAGACGGCATACGAGATCCTCTCTGGTCTCGTGGGCTCGG

HLPC purification

Nextera N709 Primer

CAAGCAGAAGACGGCATACGAGATAGCGTAGCGTCTCGTGGGCTCGG

HLPC purification

Nextera N710 Primer

CAAGCAGAAGACGGCATACGAGATCAGCCTCGGTCTCGTGGGCTCGG

HLPC purification

Nextera N711 Primer

CAAGCAGAAGACGGCATACGAGATTGCCTCTTGTCTCGTGGGCTCGG

HLPC purification

Nextera N712 Primer

CAAGCAGAAGACGGCATACGAGATTCCTCTACGTCTCGTGGGCTCGG

HLPC purification 
SCRB-seq Custom Read 1 Primer

TCTTTCCCTACACGACGCTCTTCCGATCT

HLPC purification

tSCRB Barcoded Oligo-dT Primer Plate v3

/5Biosg/ACACTCTTTCCCTACACGACGCTCTTCCGATCTJ(7)N(9)T(30)VN

/5Biosg/ denotes a 5 ' biotin; $J$ denotes a nucleic acid part of the cellular barcode; $N$ denotes a random nucleic acid part of the unique molecular identifier; V denotes A or C or G; IDT Ultramer Plate; Standard desalting

A1

/5Biosg/ACACTCTTTCCCTACACGACGCTCTTCCGATCTAACTTGGNNNNNNNNNTTTTTTTTTTTTTTTTTTTTTTTTTTTTTTTTTVN

A2

/5Biosg/ACACTCTTTCCCTACACGACGCTCTTCCGATCTCATCTGCNNNNNNNNNTTTTTTTTTTTTTTTTTTTTTTTTTTTTTTTTVN

A3

/5Biosg/ACACTCTTTCCCTACACGACGCTCTTCCGATCTAGTCACANNNNNNNNNTTTTTTTTTTTTTTTTTTTTTTTTTTTTTTTVN

A4

/5Biosg/ACACTCTTTCCCTACACGACGCTCTTCCGATCTGCTGCTTNNNNNNNNNTTTTTTTTTTTTTTTTTTTTTTTTTTTTTTTTVN

A5

/5Biosg/ACACTCTTTCCCTACACGACGCTCTTCCGATCTACTGTTGNNNNNNNNNTTTTTTTTTTTTTTTTTTTTTTTTTTTTTTTVN

A6

/5Biosg/ACACTCTTTCCCTACACGACGCTCTTCCGATCTACACTGANNNNNNNNNTTTTTTTTTTTTTTTTTTTTTTTTTTTTTTTTTVN

A7

/5Biosg/ACACTCTTTCCCTACACGACGCTCTTCCGATCTTGACGAANNNNNNNNNTTTTTTTTTTTTTTTTTTTTTTTTTTTTTTTTVN

Page 7/30 
/5Biosg/ACACTCTTTCCCTACACGACGCTCTTCCGATCTTGCACGTNNNNNNNNNTTTTTTTTTTTTTTTTTTTTTTTTTTTTTTTTVN

A9

/5Biosg/ACACTCTTTCCCTACACGACGCTCTTCCGATCTCACAAGCNNNNNNNNNTTTTTTTTTTTTTTTTTTTTTTTTTTTTTTTTTVN

A10

/5Biosg/ACACTCTTTCCCTACACGACGCTCTTCCGATCTGTATGACNNNNNNNNNTTTTTTTTTTTTTTTTTTTTTTTTTTTTTTTVN

A11

/5Biosg/ACACTCTTTCCCTACACGACGCTCTTCCGATCTAGAGCTCNNNNNNNNNTTTTTTTTTTTTTTTTTTTTTTTTTTTTTTTVN

A12

/5Biosg/ACACTCTTTCCCTACACGACGCTCTTCCGATCTCTAAGGTNNNNNNNNNTTTTTTTTTTTTTTTTTTTTTTTTTTTTTTTTVN

B1

/5Biosg/ACACTCTTTCCCTACACGACGCTCTTCCGATCTAAATGCGNNNNNNNNNTTTTTTTTTTTTTTTTTTTTTTTTTTTTTTTVN

B2

/5Biosg/ACACTCTTTCCCTACACGACGCTCTTCCGATCTGAAGCGTNNNNNNNNNTTTTTTTTTTTTTTTTTTTTTTTTTTTTTTTVN

B3

/5Biosg/ACACTCTTTCCCTACACGACGCTCTTCCGATCTGCCATATNNNNNNNNNTTTTTTTTTTTTTTTTTTTTTTTTTTTTTTTVN

B4

/5Biosg/ACACTCTTTCCCTACACGACGCTCTTCCGATCTATGCATGNNNNNNNNNTTTTTTTTTTTTTTTTTTTTTTTTTTTTTTTTTVN

B5

Page $8 / 30$ 
B6

/5Biosg/ACACTCTTTCCCTACACGACGCTCTTCCGATCTGATCCACNNNNNNNNNTTTTTTTTTTTTTTTTTTTTTTTTTTTTTTTTTVN

B7

/5Biosg/ACACTCTTTCCCTACACGACGCTCTTCCGATCTCGGTATANNNNNNNNNTTTTTTTTTTTTTTTTTTTTTTTTTTTTTTVN

B8

/5Biosg/ACACTCTTTCCCTACACGACGCTCTTCCGATCTACAGGCANNNNNNNNNTTTTTTTTTTTTTTTTTTTTTTTTTTTTTTTVN

B9

/5Biosg/ACACTCTTTCCCTACACGACGCTCTTCCGATCTGTAATGGNNNNNNNNNTTTTTTTTTTTTTTTTTTTTTTTTTTTTTTTTVN

B10

/5Biosg/ACACTCTTTCCCTACACGACGCTCTTCCGATCTAGGCTTANNNNNNNNNTTTTTTTTTTTTTTTTTTTTTTTTTTTTTTTTVN

B11

/5Biosg/ACACTCTTTCCCTACACGACGCTCTTCCGATCTGGGCAAANNNNNNNNNTTTTTTTTTTTTTTTTTTTTTTTTTTTTTTTVN

B12

/5Biosg/ACACTCTTTCCCTACACGACGCTCTTCCGATCTCTGAGACNNNNNNNNNTTTTTTTTTTTTTTTTTTTTTTTTTTTTTTTVN

C1

/5Biosg/ACACTCTTTCCCTACACGACGCTCTTCCGATCTATACACCNNNNNNNNNTTTTTTTTTTTTTTTTTTTTTTTTTTTTTTTVN

C2

/5Biosg/ACACTCTTTCCCTACACGACGCTCTTCCGATCTCGTAACGNNNNNNNNNTTTTTTTTTTTTTTTTTTTTTTTTTTTTTTTTVN 
D1

/5Biosg/ACACTCTTTCCCTACACGACGCTCTTCCGATCTTACATCGNNNNNNNNNTTTTTTTTTTTTTTTTTTTTTTTTTTTTTTTTVN

D2

/5Biosg/ACACTCTTTCCCTACACGACGCTCTTCCGATCTTCTCGTGNNNNNNNNNTTTTTTTTTTTTTTTTTTTTTTTTTTTTTTTTVN

D3

/5Biosg/ACACTCTTTCCCTACACGACGCTCTTCCGATCTCATCGAGNNNNNNNNNTTTTTTTTTTTTTTTTTTTTTTTTTTTTTTTVN

D4

/5Biosg/ACACTCTTTCCCTACACGACGCTCTTCCGATCTGGGATCTNNNNNNNNNTTTTTTTTTTTTTTTTTTTTTTTTTTTTTTTVN

D5

/5Biosg/ACACTCTTTCCCTACACGACGCTCTTCCGATCTCAGTCATNNNNNNNNNTTTTTTTTTTTTTTTTTTTTTTTTTTTTTTTTVN

D6

/5Biosg/ACACTCTTTCCCTACACGACGCTCTTCCGATCTATGAGGANNNNNNNNNTTTTTTTTTTTTTTTTTTTTTTTTTTTTTTTVN

D7

/5Biosg/ACACTCTTTCCCTACACGACGCTCTTCCGATCTCCTAAGANNNNNNNNNTTTTTTTTTTTTTTTTTTTTTTTTTTTTTTTTVN

D8

/5Biosg/ACACTCTTTCCCTACACGACGCTCTTCCGATCTCCCGAAANNNNNNNNNTTTTTTTTTTTTTTTTTTTTTTTTTTTTTTTTTVN

D9

/5Biosg/ACACTCTTTCCCTACACGACGCTCTTCCGATCTTTTGTGCNNNNNNNNNTTTTTTTTTTTTTTTTTTTTTTTTTTTTTTTVN

D10

Page $11 / 30$ 
D11

/5Biosg/ACACTCTTTCCCTACACGACGCTCTTCCGATCTCCTTCACNNNNNNNNNTTTTTTTTTTTTTTTTTTTTTTTTTTTTTTTTTVN

D12

/5Biosg/ACACTCTTTCCCTACACGACGCTCTTCCGATCTCATAGCANNNNNNNNNTTTTTTTTTTTTTTTTTTTTTTTTTTTTTTTVN

E1

/5Biosg/ACACTCTTTCCCTACACGACGCTCTTCCGATCTAGGTCAGNNNNNNNNNTTTTTTTTTTTTTTTTTTTTTTTTTTTTTTTVN

E2

/5Biosg/ACACTCTTTCCCTACACGACGCTCTTCCGATCTCTACCGANNNNNNNNNTTTTTTTTTTTTTTTTTTTTTTTTTTTTTTVN

E3

/5Biosg/ACACTCTTTCCCTACACGACGCTCTTCCGATCTTGTTCTGNNNNNNNNNTTTTTTTTTTTTTTTTTTTTTTTTTTTTTTTVN

E4

/5Biosg/ACACTCTTTCCCTACACGACGCTCTTCCGATCTACCATTCNNNNNNNNNTTTTTTTTTTTTTTTTTTTTTTTTTTTTTTTTTVN

E5

/5Biosg/ACACTCTTTCCCTACACGACGCTCTTCCGATCTCCCTCTTNNNNNNNNNTTTTTTTTTTTTTTTTTTTTTTTTTTTTTTTVN

E6

/5Biosg/ACACTCTTTCCCTACACGACGCTCTTCCGATCTGGACACTNNNNNNNNNTTTTTTTTTTTTTTTTTTTTTTTTTTTTTTTVN

E7

/5Biosg/ACACTCTTTCCCTACACGACGCTCTTCCGATCTATAGCCGNNNNNNNNNTTTTTTTTTTTTTTTTTTTTTTTTTTTTTTTVN

Page 12/30 
E12

/5Biosg/ACACTCTTTCCCTACACGACGCTCTTCCGATCTCCTGGTANNNNNNNNNTTTTTTTTTTTTTTTTTTTTTTTTTTTTTTTTVN

F1

/5Biosg/ACACTCTTTCCCTACACGACGCTCTTCCGATCTCAAGACTNNNNNNNNNTTTTTTTTTTTTTTTTTTTTTTTTTTTTTTTTVN

F2

/5Biosg/ACACTCTTTCCCTACACGACGCTCTTCCGATCTACGGGATNNNNNNNNNTTTTTTTTTTTTTTTTTTTTTTTTTTTTTTTTTVN

F3

/5Biosg/ACACTCTTTCCCTACACGACGCTCTTCCGATCTAACGGTTNNNNNNNNNTTTTTTTTTTTTTTTTTTTTTTTTTTTTTTTVN

F4

/5Biosg/ACACTCTTTCCCTACACGACGCTCTTCCGATCTTACGCCTNNNNNNNNNTTTTTTTTTTTTTTTTTTTTTTTTTTTTTTTVN

F5

/5Biosg/ACACTCTTTCCCTACACGACGCTCTTCCGATCTCACCATGNNNNNNNNNTTTTTTTTTTTTTTTTTTTTTTTTTTTTTTTTVN

Page 13/30 
F6

/5Biosg/ACACTCTTTCCCTACACGACGCTCTTCCGATCTCGAGGTTNNNNNNNNNTTTTTTTTTTTTTTTTTTTTTTTTTTTTTTTVN

F7

/5Biosg/ACACTCTTTCCCTACACGACGCTCTTCCGATCTTTCTGGANNNNNNNNNTTTTTTTTTTTTTTTTTTTTTTTTTTTTTTTTTVN

F8

/5Biosg/ACACTCTTTCCCTACACGACGCTCTTCCGATCTGGATTCGNNNNNNNNNTTTTTTTTTTTTTTTTTTTTTTTTTTTTTTTVN

F9

/5Biosg/ACACTCTTTCCCTACACGACGCTCTTCCGATCTGTTTGCGNNNNNNNNNTTTTTTTTTTTTTTTTTTTTTTTTTTTTTTTVN

F10

/5Biosg/ACACTCTTTCCCTACACGACGCTCTTCCGATCTCCACACANNNNNNNNNTTTTTTTTTTTTTTTTTTTTTTTTTTTTTTTTTVN

F11

/5Biosg/ACACTCTTTCCCTACACGACGCTCTTCCGATCTCCTTTGGNNNNNNNNNTTTTTTTTTTTTTTTTTTTTTTTTTTTTTTTTVN

F12

/5Biosg/ACACTCTTTCCCTACACGACGCTCTTCCGATCTACTACGTNNNNNNNNNTTTTTTTTTTTTTTTTTTTTTTTTTTTTTTTTVN

G1

/5Biosg/ACACTCTTTCCCTACACGACGCTCTTCCGATCTTCAACTCNNNNNNNNNTTTTTTTTTTTTTTTTTTTTTTTTTTTTTTTTVN

G2

/5Biosg/ACACTCTTTCCCTACACGACGCTCTTCCGATCTGCAATCANNNNNNNNNTTTTTTTTTTTTTTTTTTTTTTTTTTTTTTTVN

G3

Page $14 / 30$ 
G4

/5Biosg/ACACTCTTTCCCTACACGACGCTCTTCCGATCTCACGCTANNNNNNNNNTTTTTTTTTTTTTTTTTTTTTTTTTTTTTTTTTVN

G5

/5Biosg/ACACTCTTTCCCTACACGACGCTCTTCCGATCTGACGATCNNNNNNNNNTTTTTTTTTTTTTTTTTTTTTTTTTTTTTTTTVN

G6

/5Biosg/ACACTCTTTCCCTACACGACGCTCTTCCGATCTGACTCGANNNNNNNNNTTTTTTTTTTTTTTTTTTTTTTTTTTTTTTTVN

G7

/5Biosg/ACACTCTTTCCCTACACGACGCTCTTCCGATCTCGCTACTNNNNNNNNNTTTTTTTTTTTTTTTTTTTTTTTTTTTTTTTVN

G8

/5Biosg/ACACTCTTTCCCTACACGACGCTCTTCCGATCTCGTGCAANNNNNNNNNTTTTTTTTTTTTTTTTTTTTTTTTTTTTTTTVN

G9

/5Biosg/ACACTCTTTCCCTACACGACGCTCTTCCGATCTGTGTAGANNNNNNNNNTTTTTTTTTTTTTTTTTTTTTTTTTTTTTTTVN

G10

/5Biosg/ACACTCTTTCCCTACACGACGCTCTTCCGATCTACGTAGGNNNNNNNNNTTTTTTTTTTTTTTTTTTTTTTTTTTTTTTTVN

G11

/5Biosg/ACACTCTTTCCCTACACGACGCTCTTCCGATCTCGTTGGANNNNNNNNNTTTTTTTTTTTTTTTTTTTTTTTTTTTTTTTVN

G12

/5Biosg/ACACTCTTTCCCTACACGACGCTCTTCCGATCTTTAGGAGNNNNNNNNNTTTTTTTTTTTTTTTTTTTTTTTTTTTTTTTTVN

Page 15/30 
$\mathrm{H} 11$

/5Biosg/ACACTCTTTCCCTACACGACGCTCTTCCGATCTGGTTATCNNNNNNNNNTTTTTTTTTTTTTTTTTTTTTTTTTTTTTTTVN

$\mathrm{H} 12$

/5Biosg/ACACTCTTTCCCTACACGACGCTCTTCCGATCTCAAACGGNNNNNNNNNTTTTTTTTTTTTTTTTTTTTTTTTTTTTTTTTVN

\section{Equipment}

Cell sorter

Plate centrifuge

Plate sealing roller

Low temperature laboratory freezer $\left(-80^{\circ} \mathrm{C}\right)$

Laboratory freezer $\left(-20^{\circ} \mathrm{C}\right)$ and refrigerator $\left(4^{\circ} \mathrm{C}\right)$

Microcentrifuge for $1.5 \mathrm{ml}$ Eppendorf tubes

Microcentrifuge for $0.2 \mathrm{ml}$ 8-Tube Strips

Magnetic separator for 96-well plates

PCR thermocyclers (the number of cyclers equals the number of plates to be processed in parallel)

Magnetic separator for $2 \mathrm{ml}$ Eppendorf tubes

Bioanalyzer instrument

qPCR instrument

Illumina sequencer

Pipette set

Multichannel pipette set

\section{Procedure}

1) Decontaminate all contact surfaces with RNaseZap.

2) Prepare 96-well low-binding plates for single-cell sorting by dispensing $10 \mu$ l of Qiagen TCL buffer supplemented with $1 \%$ 2-mercaptoethanol per well. Seal the plates well with adhesive foil and store them at room temperature for up to one day.

(!) Do not store the plates for prolonged periods to avoid lysis evaporation. 
3) Using large nozzle size $(100 \mu)$ and single-cell purity mode, sort a single-cell of interest into each well of the PCR plate.

(!) Before sorting the population of interest, test the correct aiming of the machine. First sort 10-20 cells per well into few wells located on top and bottom of a sealed with transparent seal PCR plate. The droplets should be visible on the seal surface. Then sort 10-20 cells per well into few wells located on top and bottom of an empty PCR plate. The liquid droplet should be visible at the bottom of the PCR plate. Do not use smaller than $100 \mu$ nozzle to avoid unnecessary cell stress. The use of FACS for single-cell isolation allows for index sorting. Nevertheless, be conservative on the surface markers used for staining to avoid inducing gene expression changes.

4) Immediately after sorting, seal the plate with adhesive foil, spin it down for 30 seconds at 1000 rcf and snap-freeze it with dry ice. Transfer the plates for storage at $-80^{\circ} \mathrm{C}$ freezer.

(!) The plates can be stored at $-80^{\circ} \mathrm{C}$ for up to one year. Make sure the adhesive foil used can withstand this temperature without detaching.

5) Decontaminate all contact surfaces with RNaseZap.

6) Equilibrate the RNAClean XP beads at room temperature ( $2300 \mathrm{ml}$ per plate).

7) Prepare fresh $80 \%$ molecular grade ethanol ( $40 \mathrm{ml}$ per plate).

8) Thaw and keep the $5 \times$ Maxima H-Buffer, Advantage dNTP Mix, SCRB-seq Template Switching Oligo and tSCRB Barcoded Oligo-dT Primer Plate v3 on ice.

9) Prepare the reverse transcription component 1 (RT c1) and dispense the required amount of it into each well of a $0.2 \mathrm{ml}$ 8-tube strips with attached caps. See Table 1.

10) Prepare the reverse transcription component 3 (RT c3) and dispense the required amount of it into each well of a $0.2 \mathrm{ml}$ 8-tube strips with attached caps. See Table 2.

11) Thaw a frozen PCR plate with single cells, vortex for 5 seconds and spin down for 30 seconds at 1000 rcf. 
12) Vortex the RNAClean XP beads until fully resuspended. Dispense the beads in $25 \mathrm{ml}$ reagent reservoir. Using a multichannel pipette, add $22 \mu \mathrm{l}$ of RNAClean XP beads to each well of the single-cell plate and gently pipette 15 times to mix. Cover the plate to prevent contamination from dust and incubate for 10 minutes at room temperature.

(!) Cover the plate in a way to avoid contact between the plate and the cover. Potential contact can lead to crosscontamination. For example, the bottom of a big enough kitchen box can be used. Cover the plate whenever possible in the subsequent steps. Decontaminate the cover frequently with RNaseZap.

13) Place the plate one the magnetic separator and incubate until the solution is clear.

14) Without disturbing the ring of separated magnetic beads, aspirate and discard $20 \mu \mathrm{l}$ of the supernatant.

15) Add $180 \mu \mathrm{l}$ of $80 \%$ molecular grade ethanol to each well. If the beads are not collected in a compact ring, move the plate gently up and down the magnetic stand to collect them. Incubate for 30 seconds.

16) Without disturbing the ring of separated magnetic beads, remove the supernatant completely.

17) Add $180 \mu$ l of $80 \%$ molecular grade ethanol to each well and incubate for 30 seconds.

18) Without disturbing the ring of separated magnetic beads, remove the supernatant completely.

19) Remove any residual liquid droplets from the walls by gently tapping the magnetic stand with the plate on it to the bench surface. Aspirate the residual volume of liquid with $10 \mu \mathrm{l}$ tips loaded on a multichannel pipette.

20) Remove the PCR plate from the magnetic stand. Using a multichannel pipette, add 2,8 $\mu \mathrm{l}$ of RT component 1 to each well of the single-cell plate and mix.

(!) Time-sensitive step. Be as fast as possible. Do not allow to beads to dry and cracks to appear in the bead ring. If the magnetic stand has opposing magnetic sides (as with Thermo Fisher AM10027), you might consider resuspending four but not eight wells at a time. Magnetic stands with non-opposing magnetic sides (such as DynaMag, Thermo Fisher 12027) are easier to process. 
21) Using a multichannel pipette, add $1,2 \mu$ of the respective barcoded primer from the stock $14 \mu \mathrm{M}$ tSCRB Barcoded OligodT Primer Plate $\mathrm{v} 3$ (corresponds to reverse transcription component 2; final reaction concentration 2,4 $\mu \mathrm{M}$ ) to each well of the single-cell plate and mix.

(!) From now on, keep the plates on ice unless indicated otherwise.

(!) Before removing the transparent seal from the stock primer plate, spin the plate for 30 seconds at 1000 rcf to collect the liquid at the bottom of the wells. Be extremely careful when removing the transparent seal to avoid primer crosscontamination. In general, do not aliquot stock plates with primer for more than 20 single-cell plates. Immediately after use, reseal well the plate with transparent seal.

22) Seal the single-cell plate with adhesive foil. Incubate the plate on a thermal cycler using the following program: heated lid at $105^{\circ} \mathrm{C}, 3$ minutes at $72^{\circ} \mathrm{C}$, forever at $4^{\circ} \mathrm{C}$.

$(+)$ If you process more than one plate, this is a good moment to perform steps 11 and 12 with the next plate.

23) Spin the single-cell plate for 30 seconds at $1000 \mathrm{rcf}$ to collect the liquid at the bottom of the wells.

24) Using a multichannel pipette, add $3 \mu$ of reverse transcription component 3 (RT c3) to each well of the single-cell plate and mix.

25) Seal the single-cell plate with adhesive foil. Spin the single-cell plate for 30 seconds at 1000 rcf to collect the liquid at the bottom of the wells.

26) Incubate the plate on a thermal cycler using the following program: heated lid at $105^{\circ} \mathrm{C}, 90$ minutes at $42^{\circ} \mathrm{C}, 15$ minutes at $72^{\circ} \mathrm{C}$, hold at $4^{\circ} \mathrm{C}$.

27) Thaw and keep the KAPA HiFi HotStart ReadyMix and SCRB SMART PCR primer on ice.

(+) If you process more than one plate, you can use the incubation time to perform steps $13-26$ with the next plate. The parallel processing of more than one plate requires multiple PCR cyclers.

28) Shortly before the incubation ends, prepare the PCR master mix. See Table 3. 
29) Spin the single-cell plate for 30 seconds at 1000 rcf to collect the liquid at the bottom of the wells.

30) Dispense the necessary amount of PCR master mix in a $25 \mathrm{ml}$ reagent reservoir.

31) Using a multichannel pipette, add $18 \mu \mathrm{l}$ of PCR master to each well of the single-cell plate and mix.

32) Seal the single-cell plate with adhesive foil. Spin the single-cell plate for 30 seconds at 1000 rcf to collect the liquid at the bottom of the wells.

33) Incubate the plate on a thermal cycler using the following program: heated lid at $100^{\circ} \mathrm{C}, 3$ minutes at $98^{\circ} \mathrm{C}, 18-20 \mathrm{cycles}$ (20 seconds at $98^{\circ} \mathrm{C}, 30$ seconds at $65^{\circ} \mathrm{C}, 6$ minutes at $72^{\circ} \mathrm{C}$ ), 5 minutes at $72^{\circ} \mathrm{C}$, hold at $4^{\circ} \mathrm{C}$.

(!) The number of cycles depends on the biology of the cells used. For example, naïve T cells normally require 20 cycles, while activated T cells require 18 cycles. If different timepoints are to be compared, it is best to affix the cycles to the highest necessary number. This should not affect the performance due to the use of unique molecular identifiers, which eliminate the introduction of anPCR bias. Following this incubation, there is a potential stopping point. The plates can be stored at $4^{\circ} \mathrm{C}$ for up to 3 days before purification.

34) Equilibrate the AMPure XP beads at room temperature ( 1500 $\mathrm{ml}$ per plate).

35) Prepare fresh $80 \%$ molecular grade ethanol ( 12 $\mathrm{ml}$ per plate).

36) Spin the single-cell plate for 30 seconds at $1000 \mathrm{rcf}$ to collect the liquid at the bottom of the wells.

37) Collect barcoded single-cell transcriptomes from one plate into two $2.0 \mathrm{ml}$ DNA LoBind Tubes (columns 1-6 into one tube, columns 7-12 into another tube).

(!) The protocol allows the processing of multiple plates in parallel. The only limiting factor is the number of tube spots on the magnetic separator. 
38) Vortex the AMPure XP beads until fully resuspended. Add $870 \mu \mathrm{l}$ of AMPure XP beads to each tube and gently pipette 15 times to mix. Close the tube to prevent contamination from dust and incubate for 10 minutes at room temperature.

39) Place the tubes one the magnetic separator and incubate until the solution is clear.

40) Without disturbing the ring of separated magnetic beads, aspirate and discard the supernatant.

41) Add $2 \mathrm{ml}$ of $80 \%$ molecular grade ethanol to each tube. Incubate for 30 seconds.

42) Without disturbing the ring of separated magnetic beads, aspirate and discard the supernatant.

43) Add $2 \mathrm{ml}$ of $80 \%$ molecular grade ethanol to each tube. Incubate for 30 seconds.

44) Without disturbing the ring of separated magnetic beads, aspirate and discard the supernatant.

45) Remove any residual liquid droplets from the walls by gently tapping the magnetic stand with the tubes on it to the bench surface. Aspirate the residual volume of liquid using a $10 \mu$ tip.

46) Keeping the lids open, allow the ethanol to evaporate from the tubes until the ring of separate magnetic beads loses its glossiness (about 10 minutes).

47) Remove the tubes from the magnetic separator and resuspend the beads from each tube in $30 \mu \mathrm{l}$ molecular grade water.

48) Combine the content of the two tubes coming from one plate into a single tube and incubate for 2 minutes at room temperature.

49) Place the tube (tubes if processing more than one plate) one the magnetic separator and incubate until the solution is clear.

50) Without disturbing the ring of separated magnetic beads, transfer $55 \mu$ of the supernatant into a well of a PCR plate. 
51) Vortex the AMPure XP beads until fully resuspended. Add $33 \mu \mathrm{l}$ of AMPure XP beads to each used well of the PCR plate and gently pipette 15 times to mix. Cover the plate to prevent contamination from dust and incubate for 5 minutes at room temperature.

52) Place the plate on the magnetic separator and incubate until the solution is clear.

53) Without disturbing the ring of separated magnetic beads, aspirate and discard the supernatant.

54) Add $180 \mu \mathrm{l}$ of $80 \%$ molecular grade ethanol to each used well from the PCR plate. Incubate for 30 seconds.

55) Without disturbing the ring of separated magnetic beads, aspirate and discard the supernatant.

56) Add $180 \mu \mathrm{l}$ of $80 \%$ molecular grade ethanol to each used well from the PCR plate. Incubate for 30 seconds.

57) Without disturbing the ring of separated magnetic beads, aspirate and discard the supernatant.

58) Remove any residual liquid droplets from the walls by gently tapping the magnetic stand with the plate to the bench surface. Aspirate the residual volume of liquid using $10 \mu$ tips.

59) Allow the ethanol to evaporate from the wells until the ring of separate magnetic beads loses its glossiness (about 2 minutes).

60) Remove the plate from the magnetic separator and resuspend the beads from each well in 10-30 $\mu \mathrm{l}$ molecular grade water. Incubate for 2 minutes at room temperature.

(!) The volume depends on the cell biology. Ultimately, the final concentration should be in the quantification range of the Bionalyzer High Sensitivity DNA Kit.

61) Place the plate on the magnetic separator and incubate until the solution is clear.

62) Without disturbing the ring of separated magnetic beads, transfer $9-28 \mu \mathrm{l}$ of the supernatant into a $1.5 \mathrm{ml}$ DNA LoBind Tube. 
63) Run $1 \mu$ l of each elute on a Bionalyzer High Sensitivity chip for band quality control and quantification. See Figure 1.

(!) To run the assay follow the instructions from the manufacturer. Following this step, there is a potential stopping point. The amplified cDNA can be stored at $-20^{\circ} \mathrm{C}$ for several months.

64) Thaw and keep the Tagment DNA Buffer (TD buffer), Amplicon Tagment Mix (ATM), Nextera PCR Master Mix (NPM), 10 $\mu \mathrm{M}$ SCRB-seq custom N5 primer and $10 \mu \mathrm{M}$ Nextera P7 index primers on ice.

(!) The use Nextera XT N5 index primer is substituted with SCRB-seq custom N5 primer, which is used along with Nextera XT N7 index primer. As the SCRB-seq custom N5 primer is universal for all plates to be pooled during sequencing, the indexing is introduced with the use of different Nextera XT N7 primers (e.g. N701-N712).

(!) Perform steps 62 - 78 on ice if not stated otherwise.

65) Dilute $1 \mathrm{ng}$ of amplicon from each single-cell plate to a volume of $5 \mu \mathrm{l}$ in a well of a PCR plate.

66) Add $10 \mu$ TD buffer to each used well of the PCR plate.

67) Add $5 \mu$ ATM to each used well of the PCR plate.

68) Using a multichannel pipette set to $15 \mu \mathrm{l}$, gently mix the reaction 10 times.

69) Seal the PCR plate with transparent seal. Spin the plate for 30 seconds at $1000 \mathrm{rcf}$ to collect the liquid at the bottom of the wells.

70) Incubate the plate on a thermal cycler using the following program: heated lid at $100^{\circ} \mathrm{C}, 5$ minutes at $55^{\circ} \mathrm{C}$, hold at $4^{\circ} \mathrm{C}$.

71) Immediately after incubation, add $5 \mu$ I Neutralize Tagment Buffer (NT buffer) to each used well of the PCR plate and gently mix 10 times. 
(!) As following the incubation the transposase is still active, it is critical to perform step 71 in timely manner.

72) Cover the plate to prevent dust contamination and incubate for 5 minutes at room temperature.

73) Add $15 \mu$ I NPM and $8 \mu \mathrm{l}$ molecular grade water to each used well of the PCR plate.

74) Add $1 \mu \mathrm{l}$ of the $10 \mu \mathrm{M}$ SCRB-seq custom N5 primer to each used well of the PCR plate.

75) Add $1 \mu \mathrm{l}$ of the respective indexing $10 \mu \mathrm{M}$ Nextera P7 primer to each sample.

76) Using a multichannel pipette set to $30 \mu \mathrm{l}$, gently mix the reaction 10 times.

77) Seal the PCR plate with transparent seal. Spin the plate for 30 seconds at $1000 \mathrm{rcf}$ to collect the liquid at the bottom of the wells.

78) Incubate the plate on a thermal cycler using the following program: heated lid at $100^{\circ} \mathrm{C}, 3$ minutes at $72^{\circ} \mathrm{C}, 30$ seconds at $95^{\circ} \mathrm{C}, 12$ cycles $\left(10\right.$ seconds at $95^{\circ} \mathrm{C}, 30$ seconds at $55^{\circ} \mathrm{C}, 30$ seconds at $\left.72^{\circ} \mathrm{C}\right), 5$ minutes at $72^{\circ} \mathrm{C}$, hold at $10^{\circ} \mathrm{C}$.

(!) Following this step, there is a potential stopping point. The plate can be left overnight in the thermal cycler or stored for up to two days at $4^{\circ} \mathrm{C}$.

79) Equilibrate the AMPure XP beads at room temperature ( 60 $\mu$ l per sample).

80) Prepare fresh $80 \%$ molecular grade ethanol ( 800 $\mu$ l per sample).

81) Spin the plate for 30 seconds at $1000 \mathrm{rcf}$ to collect the liquid at the bottom of the wells.

82) Vortex the AMPure XP beads until fully resuspended. Add $30 \mu$ of AMPure XP beads to each used well of the PCR plate and gently pipette 15 times to mix. Cover the plate to prevent contamination from dust and incubate for 5 minutes at room temperature. 
83) Place the plate on the magnetic separator and incubate until the solution is clear.

84) Without disturbing the ring of separated magnetic beads, aspirate and discard the supernatant.

85) Add $180 \mu \mathrm{l}$ of $80 \%$ molecular grade ethanol to each used well from the PCR plate. Incubate for 30 seconds.

86) Without disturbing the ring of separated magnetic beads, aspirate and discard the supernatant.

87) Add $180 \mu \mathrm{l}$ of $80 \%$ molecular grade ethanol to each used well from the PCR plate. Incubate for 30 seconds.

88) Without disturbing the ring of separated magnetic beads, aspirate and discard the supernatant.

89) Remove any residual liquid droplets from the walls by gently tapping the magnetic stand with the plate to the bench surface. Aspirate the residual volume of liquid using $10 \mu$ tips.

90) Allow the ethanol to evaporate from the wells until the ring of separate magnetic beads loses its glossiness (about 2 minutes).

91) Remove the plate from the magnetic separator and resuspend the beads from each well in $52 \mu \mathrm{l}$ molecular grade water. Incubate for 2 minutes at room temperature.

92) Place the plate on the magnetic separator and incubate until the solution is clear.

93) Without disturbing the ring of separated magnetic beads, transfer $50 \mu$ l of the supernatant into a well of a new PCR plate.

94) Vortex the AMPure XP beads until fully resuspended. Add $30 \mu$ of AMPure XP beads to each used well of the new PCR plate and gently pipette 15 times to mix. Cover the plate to prevent contamination from dust and incubate for 5 minutes at room temperature.

95) Place the plate on the magnetic separator and incubate until the solution is clear. 
96) Without disturbing the ring of separated magnetic beads, aspirate and discard the supernatant.

97) Add $180 \mu \mathrm{l}$ of $80 \%$ molecular grade ethanol to each used well from the PCR plate. Incubate for 30 seconds.

98) Without disturbing the ring of separated magnetic beads, aspirate and discard the supernatant.

99) Add $180 \mu \mathrm{l}$ of $80 \%$ molecular grade ethanol to each used well from the PCR plate. Incubate for 30 seconds.

100) Without disturbing the ring of separated magnetic beads, aspirate and discard the supernatant.

101) Remove any residual liquid droplets from the walls by gently tapping the magnetic stand with the plate to the bench surface. Aspirate the residual volume of liquid using $10 \mu$ tips.

102) Allow the ethanol to evaporate from the wells until the ring of separate magnetic beads loses its glossiness (about 2 minutes).

103) Remove the plate from the magnetic separator and resuspend the beads from each well in $10 \mu \mathrm{l}$ molecular grade water. Incubate for 2 minutes at room temperature.

104) Place the plate on the magnetic separator and incubate until the solution is clear.

105) Without disturbing the ring of separated magnetic beads, transfer $9 \mu$ l of the supernatant into a PCR tube.

106) Run $1 \mu$ l of each elute on a Bionalyzer High Sensitivity chip for band quality control. See Figure 2.

(!) To run the assay follow the instructions from the manufacturer. Following this step, there is a potential stopping point. The libraries can be stored at $-20^{\circ} \mathrm{C}$ for several weeks. Bioanalyzer can not be used as a mean for library quantification. The library quantification and pooling must be performed based on the Illumina recommendations (SY-930-1010, Illumina) with the use of KAPA SYBR FAST qPCR Master Mix (KK4600, Kapa Biosystems).

\section{Sequencing Recommendation:}


Sequencing read 1 (SCRB-seq Custom Read 1 Primer): 16 bp

Index read 1 (Illumina HP12 primer): 8 bp

Sequencing read 2 (Illumina HP11 primer): 50 - 100 bp

* The Illumina HP10 read 1 primer must be replaced with SCRB-seq Custom Read 1 Primer. For replacement, follow the Illumina recommendations.

** Aim for $0.5-1$ million reads per cell (48 - 96 million reads per 96-well single-cell plate)

\section{Troubleshooting}

\section{Time Taken}

\section{Anticipated Results}

\section{References}

1 Soumillon, M., Cacchiarelli, D., Semrau, S., van Oudenaarden, A. \& Mikkelsen, T. S. Characterization of directed differentiation by high-throughput single-cell RNA-Seq. bioRxiv, 003236, doi:10.1101/003236 (2014).

2 Kanev, K. et al. Proliferation-competent Tcf1+ CD8 T cells in dysfunctional populations are CD4 T cell help independent. Proceedings of the National Academy of Sciences 116, 20070-20076, doi:10.1073/pnas.1902701116 (2019).

\section{Acknowledgements}

The work was supported by the European Research Council starting and consolidator grants (ProtecTC, and ToCCaTa) and from the German Research Foundation (SFB1054).

\section{Tables}

Due to technical limitations, Tables 1-3 can be found in the Supplementary Files section

\section{Figures}


Figure 1

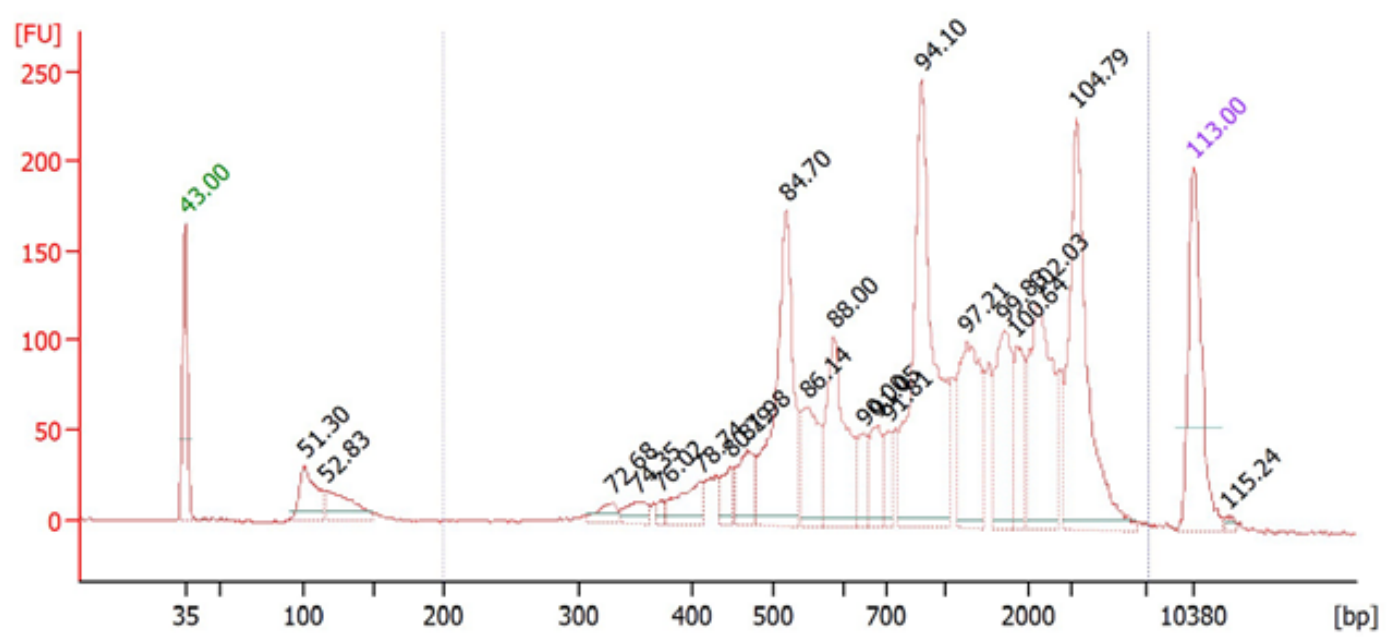

Figure 1

Typical amplicon profile of a single-cell plate with T cells following 20 cycles of cDNA amplification.

\section{Figure 2}

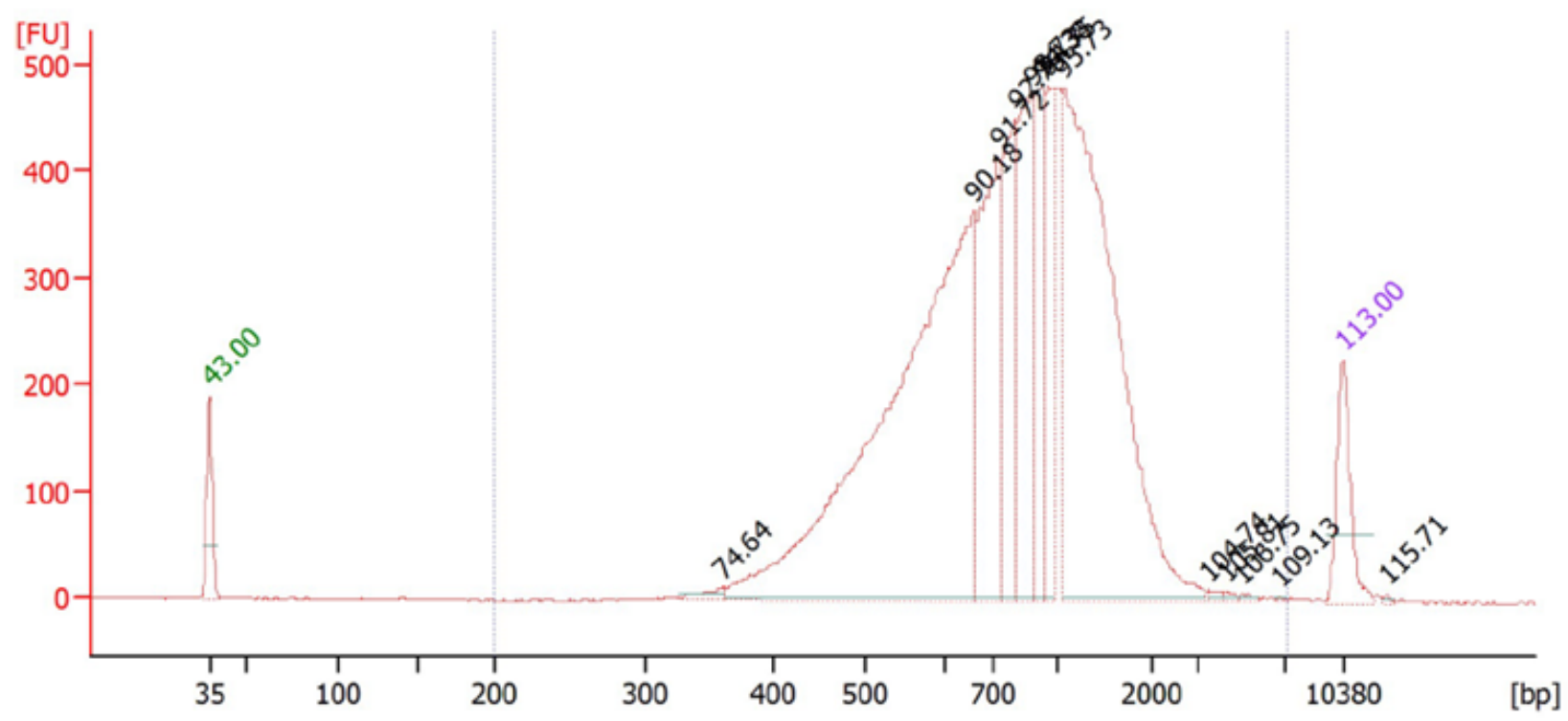

Figure 2

Typical library profile of a single-cell plate with T cells.

\section{Supplementary Files}

This is a list of supplementary files associated with this preprint. Click to download.

- tSCRBBarcodedOligodTPrimerPlatev3sequences.xls

- Table3.png

- Table1.png 
- Table2.png

Page 30/30 